\title{
Atypical development spectra considering the hunter-breeder culture transition - Spectra of the atypical neural development
}

Gyarmathy Éva, Institute of Cognitive Neuroscience and Psychology of the Research Centre for Natural Sciences

Jennifer Plosz, Visualization and Mathematics, University of Calgary

\section{Introduction}

According to Merlin Donald (1991), the evolution of Homo sapiens has been greatly influenced by communication abilities, such as speech, literacy, and now the newest technological tools and media, which in turn has led us to a new stage for the species. with the increased usage of communication technology, this direction of change has become even more reinforced. Technology is progressing exponentially (Kurzweil, 2005), and as a consequence, the transformation of the human brain has also accelerated.

The change in neurological development brought about by the changes in the physical, biological, and psychological stimulus from the environment is especially pronounced in small children.

Yet, it is evident that our social sciences, including psychology and pedagogy, have fallen behind in terms of teaching methods. Currently, our society is grown into a much more image-based, visual, and technological culture, yet this shift does not appear to have occurred in psychology and pedagogy. Meaning that the portion of our society, which is meant to support our children has abandoned them to a medium that is transforming in an extremely rapid and significant way. Why are there more and more atypical neurological development? How does the change of the culture impact the atypical learners? In what ways is our evolving culture improving their experience and learning and in which ways is it further hindering?

\section{ATYPICAL DEVELOPMENT}

\section{Our cognitive fingerprint}

No two brains are alike, yet it cannot be denied that with diversity there are also trends and similarities. Each of us has a unique brain fingerprint. Just as fingerprints have their own unique pattern made up of grooves and ridges, so do our brains. Yet, even our very unique fingerprints can be categorized based on similar patterns; there are three basic overarching fingerprint designs: Whorl, Arch, and Loop. In the study of brain physiology, we can likewise make similar categorizations to help us notice some broader trends.

If we look at the physiology of our hands, they are similar, but no two hands are exactly alike. If we focus attention on the width and length of our fingers, some of us have quite short stubby fingers, some have very long slender fingers, and the rest of us lie somewhere in between. The structure of our hands can have an impact on the types of tasks we are more suited towards. Delicate slender fingers may be more suited to precision, detail work, whereas, strong wide fingers may be more suited to strength-based tasks. Just as some 
fingers are wide and some narrow, so it is with our brains, some brains are made up of wider folds, some have more narrow folds, and the rest fall somewhere in between. These distinctions may cause certain brain structures to be more suited to particular abilities and tasks. As we consider these structural differences, it may be productive to use Morton and Frith's (1995) theoretical approach by discussing the behavior level (directly observable), the cognitive level (memory, language, and processing speed), and then the brain level (neural structures and process). However, we will explore them in the reverse order as structure, may impact cognition and then cognition would impact behaviour.

\section{The Brain Level}

Looking at the brain's structural level, for example Casanova and his colleagues have hypothesized that two cognitive profiles stand at either end of a spectrum from each other (Casanova, et al, 2002; Casanova, et al. 2004; Casanova, et al. 2010; Williams \& Casanova, 2010). Casanova's theories arise from research into the brain structure of those with dyslexia or autism. It is through this research that Casanova (Casanova, et al. 2004; Casanova, et al. 2010;) explores such elements as gyrification and neural connectivity. What they found was that dyslexics have less gyrification and wider gyral windows than "typicals", whereas those with autism were found to have more gyrification and narrower gyral windows than their more typical counterparts - wider folds versus narrower folds. This increase in folds causing the folds to be narrower, possibly as a result, the white matter area held within the fold is denser than in a "typical" brain. This then may affect how their neural connections are created.

Autistic brain structures create more local connections than a "typical" brain structure and fewer long-range connections which also causes them to have fewer connections between the two hemispheres of the brain than "typicals". The dyslexic brain, on the other hand, has fewer folds than the "typical" brain. This decrease in folds causes the folds to be wider, which makes the white matter area held within the fold more spacious. This affects how their neural connections are created. Dyslexic brain structures create more long-range connections than a "typical" brain structure and fewer local connections. More long-range connections also cause them to have more connections between the two hemispheres of the brain than "typicals". It is through these structural differences that the hypothesis of autism being at one end of a spectrum and dyslexia at the other end, with what may be considered "neurotypicals" falling somewhere in-between.

The neural system approach, proposed by Nicolson and Fawcett (2007), provides an intermediary level between the brain and cognition that is appropriate for the analysis of learning skills and disabilities. The neural system is close enough to the cognitive processes and skills underlying performance to support investigations of behaviour and education and it is close enough to the underlying brain mechanisms to support investigations of cognitive neuroscience.

\section{The Cognitive Level}

These structural distinctions will no doubt affect how these individuals think and learn.

People with specific learning difficulties (dyslexia, dyspraxia, dyscalculia) are also called "3D" people, which is a perfect term. On a cognitive level one of their main problem is that they 
miss the third dimension in the literal world, in the school-books, on the screens. They function properly in the real world, in the 3D space. They are discussed to be the bigger picture, conceptual thinkers, strong visual-spatial, and good at making connections that others may struggle with (Eide \& Eide, 2011). Research has also discussed weak neural adaptation (Perrachione, et al, 2016) and similarly the automatization deficit hypothesis (Nicolson, \& Fawcett, 1990) in terms of areas of struggle. Whereas those on the autism spectrum, tend to be very detail-oriented and strong memorizers, yet both atypical groups tend to be slow processors.

\section{The Behaviour Level}

Williams and Casanova (2010) further hypothesized that in regards to our general populous some people may present closer to the autistic end of the spectrum, as they tend towards being more detail-oriented and develop very specialized skills, crave routine, and excel at rote memorizing, but may struggle to make some of the larger or big picture connections. This may cause them to struggle at school by missing the main objective of an assigned project and instead focus on a small detail and get off course. Or they may struggle to complete tasks as they become fixated on the details. Yet, they may excel at school tasks that involve rote memorization. A person with autism often struggles to see the forest for the trees.

Those with an opposite brain structure tend towards a 3D way of thinking and therefore behaving. 3D people tend to be very creative through their ability to make connections between ideas that others may not, but they struggle to learn the details. As a result, 3D children can struggle in elementary school with all the many skills that require absorption of details and automaticity, such as reading, writing, and often mathematics, depending on how it is taught. They tend to crave difference and creativity. They struggle to see the trees for the forest, but if they are not given the forest/big picture/conceptual aspect of a topic they are often left without a starting point, which is the root of a lot of their struggle with school.

\section{The group of atypical development spectrum}

While researchers identified dyslexia and autism as the ends of a spectrum, where the ends are the atypical brains, and the fewer extremes are the typical brains, dyslexia, dysgraphia, dyspraxia, dyscalculia, attention deficit, hyperactivity, and autism spectrum disorder are not entirely separate deviations, but instead have common bases in special neurological characteristics. Their neurological kinship is also indicated by their extremely frequent concurrence in various combinations.

According to the studies of Richardson and Ross (2000), the cause behind this frequent comorbidity is a set of abnormalities in the production of fatty acids which play an important role in neural transfer in the brain.

Research results have shown that movement planning and coordination, seriality, as well as the ability to keep rhythm, are problem areas in the case of dyslexia, attention deficit, and hyperactivity disorder, and autism spectrum disorder, alike, and the same applies to a deficit in inhibition and implementation functions, which play an important role in control (Schonfeld, et al, 1989; Denckla, et al, 1985; Barkley, 1997; Piek et al, 1999; Greenspan \& 
Wieder, 1999). It has long been evident that speech sound processing difficulties feature in the development of dyslexia (see, e.g., Pennington et al, 1990), but a poor and unreliable processing of speech sounds is also often a characteristic in the case of autism spectrum disorder (see, e.g., Bomba, Pang, 2004; Bishop, Snowling, 2004).

Less definitive hand preference is more often found in autism spectrum disorders as opposed to neurotypical children (e.g., Knaus, Kamps and Foundas, 2016; Preslar, Kushner, Marino and Pearce, 2014) and also atypical or weak cerebral lateralization and handedness is associated with neurodevelopmental disorders such as dyslexia (e.g., Bishop, 2013; Vlachos, 2013). According to Pauc (2005), concurrences are so frequent that we could identify the syndrome jointly as late development syndrome. Robert Melillo (2009) used the term "Disconnected Kids".

All of these deviations that are identified as disorders may, in many respects, form the basis of gifted development, too, and so gifted development also belongs to atypical developments (Gyarmathy, 2009; Mrazik, Dombrowski, 2010). This indicates that an atypically developed nervous system may have performance advantages, as well, that is, there is also some evolutionary benefit linked to deviation. The concurrence of deviations diagnosed as disorders with each other and with gifted development, alike, as well as their shared neurological background processes are indicative of mutually overlapping developmental exceptionalities.

\section{Different forms of atypical development}

Atypical development affects those parts of the cognitive system which are affected by the maturing of the nervous system. The shared neurological characteristics behind different forms of atypical development are responsible for shaping the basis of the syndrome. At the same time, internal and external factors can contribute to a manifestation of cognitive deviations in reading, counting, literacy, attention and behaviour control, the concreteness of perception and information processing, as well as outstanding cognitive achievements in different combinations and with different weights (Figure 1).

Usually, autism is described as a spectrum disorder, which means that the syndrome can appear in very different levels and forms. However, the same is true for learning difficulties and control disorders, even for giftedness. So, we call them spectra.

Figure 1. Forms of atypical development

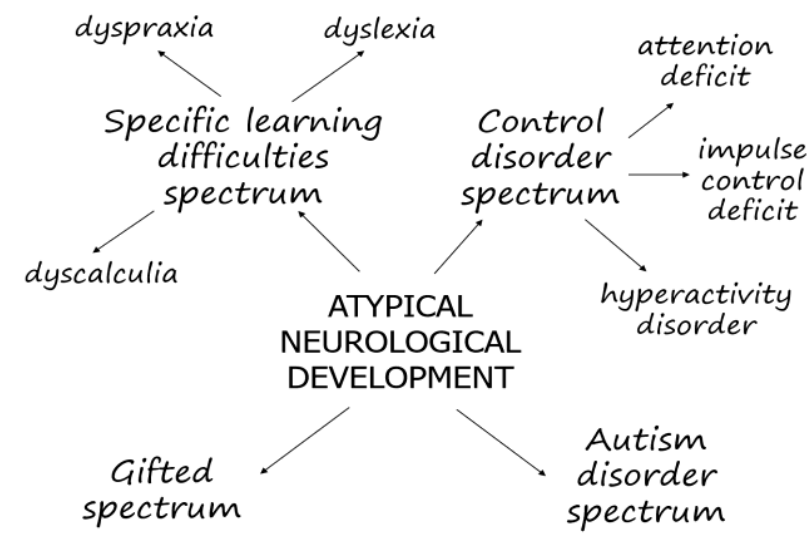


All forms of atypical development are characterized by the following:

- it is based on a different form of normal neurological functioning;

- it has advantages and disadvantages throughout the individual's lifetime;

- it is independent of intelligence;

- it is environment-dependent.

The last feature explains why the manifestation of atypical development has been heavily affected by the changes in the $21^{\text {st }}$ century. The increased prevalence which may be in part due to environmental factors of each of these exceptionalities has come to receive special attention, as has their study. On the other hand, also the impact of former cultural changes should be considered in the understanding of atypical neurological development.

\section{CULTURAL CHANGES AFFECT THE BRAIN DEVELOPMENT}

\section{The fruits of the brief dalliance of Homo sapiens with literacy}

About 2.5 million years ago, the more "hyperactive" apes, who were too restless to sit around, climbed down from the trees and started running around on the ground, and then as a result began to develop more strongly, and to think and communicate. They developed new cognitive functions for joint hunts. Prehistoric men even learned how to ignite a fire a million years ago (Gowlett, 2016). Homo sapiens are a relatively new phenomenon, it is only about 100000 years old but have become a veritable success.

Humanity undertook a significant cultural change at the end of what is called the Palaeolithic age, and instead of relying on the uncertain methods of hunting and gathering, invented agriculture. With the appearance of animal husbandry and cultivation, the Neolithic as a new culture was a turning point in the development of the human brain, as well. Methodical, temporally segmented work came to replace the rather unpredictable, and hence trial-anderror-based hunter-gatherer lifestyle. This change also came to manifest itself in the nervous system in the few thousand years of the agricultural lifestyle becoming widespread. So successful was the methodical work that the human population quickly started growing, and in the meantime, it also laid down the neurological bases of literacy (Diamond \& Bellwood, 2003).

Figure 2. Cultural changes in a sketch

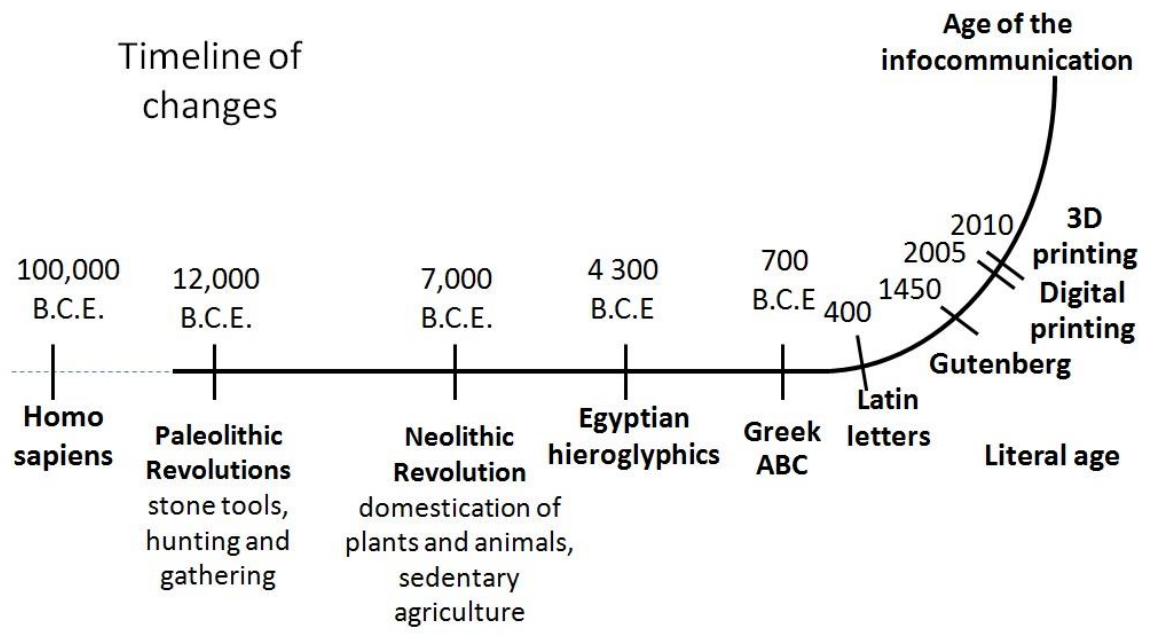


The Neolithic came to an end with the appearance of literacy, and the historical age began. The brain functions of humanity, suited to its hunter-gatherer activities, underwent significant changes by turning to an agricultural lifestyle requiring method, planning, and foresight (Figure 2).

The perception and activities of a nomad hunter-gatherer can be characterized by the following:

- spatial, visual, impulsive, cooperative

- not efficient at storing, but instead acquires

- seeks, finds, chooses

- hunts, explore, collects, moves on

- conjectures, imagines, intuits

- risk-taking and trial-and-error

The perception and activities of a settled breeder can be characterized by the following:

- time-bound, delimited, controlled, sequential

- sows, tends, harvests, stores

- foresighted, economical, methodical

- stationary, regulated work

- plans, methodically implements

- methodical thinking

The human brain was used for a nomad, hunter-gatherer lifestyle for 2 million years, while neurological processes required by an agricultural lifestyle count as very new and young from an evolutionary point of view. All the functions that form the basis for literacy are formed during the preparation for activities that are connected with culture. This process is called the maturation of the nervous system, during which a child basically with a brain of a "hunter-gatherer" gradually becomes suited for literacy and, ultimately, literate.

Anthropological research suggested that changes in the human language supported the developing agricultural mind to understand relations. The analysis of the languages of the still exiting hunter-gatherer societies shows a grammar so much different from most of the languages, that even the so-called inner "language acquisition device" or universal grammar theory is questioned (Everett, 2005). For example, Pirahă is a language isolated in the Brazilian Amazon, that lacks markers of subordination, coordinating conjunctive and disjunctive particles, as well as syntactic complement clauses. Pirahă grammar does not allow recursive possession, multiple modifications in the same phrase, etc. (Everett, 2005; 2012).

Grammar mirrors the way of information processing, and in the example of the Pirahă language, it is clear that their brains don't use the relations that are not important in their culture, but is relevant in agricultural and industrial ones like most of the cultures more recently dominating the Earth.

It is not only reading that is found to be an unnatural basic ability of the Homo sapiens but also counting. Numbers do not exist in all cultures. A small portion of the world's languages 
is anumeric or nearly anumeric. To this day, there are numberless hunter-gatherers deep in Amazonia and Australia. Instead of using words for precise quantities, these people rely on terms analogous to "a few" or "some" indicating that number words are not a human universal, but a result of cultural changes (Gordon, 2004; Zhou \& Bowern, 2015). The language for an exact number is a cultural invention rather than a universality, and number words are a cognitive technology for keeping track of the cardinality of large sets across time, space, and changes in modality (Frank et al, 2008).

Research on Australian Aborigine people demonstrates a cross-cultural difference in thought that is more than a matter of style or preference. Instead of words like "right," "left," "forward," and "back," which, define space relative to an observer, Pormpuraawans, like many other Aboriginal groups use cardinal-direction terms (north, south, east, west) to define space, and they do the same for time. These findings show that conceptions of even such fundamental domains as time can differ dramatically across cultures. Cross-cultural differences in basic spatial representations may have far-reaching consequences for other knowledge domains in the cognitive system. (Gaby, 2011; Boroditsky \& Gaby, 2010).

Reading, writing, and counting became essential knowledge for an agricultural lifestyle, and then more and more information communication activities started building on these skills. School education serves to prepare children for methodical work. An agricultural lifestyle requires a brain which

- can carry out routine tasks,

- knows the process beforehand and plays safe,

- and great professional knowledge is its forte.

In contrast, in ancient hunter-gatherer cultures, humanity found the kind of brain useful for a million years which

- is quick to respond to situations,

- can adapt to an unknown future,

- problem-solving ability is its forte.

Already at first glance, it is evident that to attain good achievements, it's best to have both kinds of thinking, but in general, it is task-depend, how much of each is needed. The latter is the leading approach in hunting, while the former in agriculture. Different eras differ in this respect.

By the $21^{\text {st }}$ century, progress in information communication has reached a level that is leading to a culture of change. In the age of literacy, knowledge had to be planted, grown, harvested, and stored like crops. In the information age, it has become possible to acquire information through "hunting and gathering", and those who use this method become more successful than those who continue to choose the lengthy process of knowledge cultivation. In other words, a former, older way of functioning has again become useful.

The cerebral functions of our nomad hunter-gatherer ancestors can once again be advantageous. At the same time, all that the methodical, agricultural, literacy-based approach developed in the human brain still remains indispensable, and not only at school: methodical thinking also forms part of the background of higher-level, synthesizing, and critical thinking. 


\section{Physical and biological environmental effects}

The cerebral functions that are newer from an evolutionary point of view are less stable, and as such are more susceptible to environmental influences. Humans come into contact with diverse kinds of toxic substances, viruses impact the brain, which their internal defence system is more or less capable of neutralizing. However, not every brain is always able to cope with the increased and unnatural strain on their system successfully. Predisposing and triggering factors mutually define the impact.

There is already a long history of research on the connection between food containing synthetic materials and developmental disorders (see, e.g., Feingold, 1974; Rowe, 1988). Jane Healy in her book, Endangered Minds (1990), explores the poisons of modern civilization. One of these everyday poisons, the sweetener called Aspartame, contains phenyl-alanine, which is responsible for phenylketonuria. This compound can pass through the blood-brain barrier and when it has accumulated in a large quantity, it leads to neurological disorders (Healy, 1990).

The "Gut and Psychology Syndrome" could play a role in the appearance of learning-, hyperactivity-, attention- and autism spectrum disorders, as well as depression and schizophrenia (Campbell-McBride, 2005; 2010). Antibiotics ruin the gut flora, because of which the gastrointestinal tract produces toxic substances, which are then transmitted to the brain. At birth, mothers might pass on their abnormal intestinal bacteria to their children, which can lead to the development of harmful intestinal flora and early brain poisoning starting from infancy. This has increased the risk of developmental disorders in children with sensitivity (Ward, 2001).

Environmental triggers such as industrial and urban pollution, mercury and other chemicals induce atypical development (for example Matsuzaki, et al, 2012; Kouichi Yoshimasu et al, 2015).

Mobile phones and other artificial radiation sources, as well as the increasing number of electronic gadgets, which generate magnetic fields, have a physiological effect on brain development (see, e.g., Cotgreave, 2005; Ferreri, et al. 2006).

These frequent, mildly injurious environmental effects can lead to mild brain trauma that could cause impairment of brain functions described earlier as minimal brain (cerebral) dysfunction which is a now obsolete considered term, used in the early 1960s (Rosa, 2010).

The trauma affects the area of the brain associated with perception, behaviour, and academic ability. The usually mild dysfunction influences one or more of the basic psychological processes involved in ADHD, understanding or in using spoken or written language, and is characterized by specific learning difficulties. Certainly, the mild brain trauma that was named 'minimal brain or cerebral dysfunction' causes disturbances at the newer, less basic functions of the developing nervous system, which is exactly what appeared only about ten thousand years ago, namely the functions needed for the farmer lifestyle.

Recent genetic evidence shows that identical genetic causes are common among different neurodevelopmental disorders. Findings provide strong evidence in support of not only reviving the old concept of minimal brain (cerebral) dysfunction but also expanding the scope to include a broad spectrum of neurodevelopmental disorders Developmental brain 
dysfunction, whether genetic or caused by an insult to the developing central nervous system, such as exposure to a teratogen, trauma, infection, severe nutritional deficiency, or hypoxia-ischemia, is typically manifested as impairments in cognitive, neuromotor, or neurobehavioral functioning. It results in clinical manifestations that include disorders once encompassed by minimal brain dysfunction or minimal cerebral dysfunction (e.g., learning difficulties, language disorders, developmental coordination disorder, and ADHD), and the classic neurodevelopmental disabilities (e.g., intellectual disability, cerebral palsy, and autism spectrum disorders) (Moreno-De-Luca et al, 2013).

Specific genetic causes, including certain copy number variants and single-gene mutations, are shared among disorders that are thought to be clinically distinct. This evidence of variability in the clinical manifestations of individual genetic variants and sharing of genetic causes among clinically distinct brain disorders is consistent with the concept of developmental brain dysfunction, a term Moreno-De Luca et al use to describe the abnormal brain function underlying a group of neurodevelopmental and neuropsychiatric disorders and to encompass a subset of various clinical diagnoses.

Different human bodies and brains depending on their genetic backgrounds react to harmful effects differently. In some people, even a small amount of exposure has a strong influence on cerebral functions, while there is no perceptible effect in others.

\section{Atypical development is a deviation in neurological maturation}

We consider that at least three, mutually independent, but cooperative systems are involved in the background of all cognitive achievements. We must consider them separately when analysing:

1. The maturing of the nervous system, the characteristics of the neurological system, typical or atypical neural functioning

2. Intelligence, the level of thinking, and learning efficiency

3. Abilities (linguistic, musical, visual, kinaesthetic, etc.), the bases for thinking and learning, which form an individual-specific system of abilities

According to our concept, the different forms of the neurologically-based achievement difficulties are a delayed or atypical development of a brain changing from a nomad into a farmer. The neurological functions that formed the basis of literacy for humanity, and which from a developmental point of view can be regarded as fairly novel, are those which embody school-readiness in small children:

- has developed toleration of monotony, can sit still,

- can focus its attention, maintain it for at least 15 minutes, can remember sequential pieces of information,

- can control its urges and wait patiently,

- its perception is refined, can identify details and relations,

- its gross and fine motor movements are coordinated and able to maintain rhythm. 
The core of being a good learner at school is the well-working executive functions (EF). EF refers to the ability to plan, follow instructions, and methodically carry out an activity without distraction. Less effective executive functions have been identified in attention deficit hyperactivity disorder (Diamond, 2013) and autism (Solomon, 2007), as well.

It is not difficult to identify the agricultural or its more developed descendant the industrial cultured brain behind the well-working executive functions. The essence of this functioning is to follow the row from A to B while avoiding distraction. Though even psychologist can't agree exactly which functions belong to the executive functions, there is a concord that three groups of functions can be identified:

1. Self-control, including behavioral inhibition, supports setting priorities and resisting impulsive actions or responses

2. Mental flexibility helps to sustain or shifting attention, interference control (selective attention and cognitive inhibition)

3. Working memory governs the ability to retain and manipulate distinct pieces of information over short periods

The three types of atypical neurological development are based on the deviations in the same neural networks but differ from each other mildly by the way these networks are involved. In terms of the executive function groups, mainly self-control dysfunctions can be identified behind the control disorder spectrum, mental flexibility is a problem of the autism disorder spectrum and the unstable working memory is a feature of learning difficulties.

Executive functions have been consciously trained since the early agricultural societies appeared. The best examples are the old East Asian cultures, where various types of arts, board games, motion, and mind control like Thai Chi or meditation, and even juggling were and still are everyday activities.

While executive functions are needed in our everyday life, they were not so important in a hunter-gatherer world. It is more advantageous for a hunter-gatherer to pay attention to every little detail in their surroundings and hyper-focus when the target is found. It is less possible to plan and execute a given activity in such a situation. You plan to find blueberries, but it is important to move on from your plan and allow yourself to be distracted by the delicious and nutritious mushrooms you encounter along your travels.

In contradiction to the industrial $20^{\text {th }}$ century, the info-communicational $21^{\text {st }}$ century needs minds that can both execute and deviate. Atypically developing brains are advantageous rather in deviation than in execution, and dyslexic people can be very successful, once they have survived the old-fashioned school and get to a basic level of the executive functions. See, for example, Positive Dyslexia (Nicolson, 2015) or the atypically developing gifted persons (Gyarmathy, 2009).

Even the so-called Delayed Neural Commitment (DNC) is not only a disadvantage but a way to build a more conscious approach (Nicolson \& Fawcett, 2019). DNC is a feature of the dyslexic brain, which can be responsible for the delay in the development of specific skills and automaticity. Delays both in constructing new neural circuits and in bypassing or eliminating the previous, less efficient neural circuits. This is an adaptive neurological functioning in a nomad lifestyle in a changing environment when one needs very few 
important, stable, repetitive skills to be effective, and it is not worth it to store temporary information. A superficial information processing and an ability to forget may be a useful approach in the $21^{\text {st }}$ century's fast-changing world. It is more economical not to build strong neural connections, rather merely perceive and process the stimuli consciously.

The early agricultural societies and those that have followed them, for ten thousand years developed the abilities that make a proper farmer or in more recent past a good industrial worker, which means to adapt the neurological system to a stable environment, where it is important to learn effectively the skills and store information that will be useful in one's entire life.

However, by the $21^{\text {st }}$ century, the appearance of increasingly more highly developed technological tools, and in particular, the appearance of information communication technology has transformed daily life. Everyday experience necessary for 'farmer lifestyle' has diminished:

- children are surrounded by an increased amount, of changing stimuli,

- their brain is subject to an increased amount, of stimuli, which strengthen the shortterm memory system but leads to poor attention,

- they do not have to restrain themselves in the same way (washing machines clean everything, plastic tools do not break easily, lost objects can be replaced, etc.),

- they receive more holistic, visual stimuli and are confronted with fewer situations requiring finely-tuned, verbal processing,

- there are less movement, information, and experience that can be acquired with less activity, there are less precision and manual activities, and possibly less sensorymotor experience.

If we compare all this with the requirements of school readiness, we see point by point the environmental influences slowing down the maturation of our methodological thinking of our agricultural-literal brain.

Everyday life and care have adapted to technological changes, and so children have a far greater opportunity to explore the information space and satisfy their curiosity. At the same time, activities that prepare for literacy have fallen into the background. There is less reading aloud, more moving pictures, less active usage of music instruments, more listening to music, less physical-bodily experience, more mental experience.

The culture that wires children's brains has changed in several ways, and this causes lags in neurological development in different children to different extents. The conversion of humanity to the "agricultural" brain functions and literacy took effect to different degrees genetically in different individuals and groups. There have always been individual differences in this respect, and there have always been people, who lagged in terms of literacy, or who reacted to environmental stimuli more sensitively. These are the first who fall into the group of atypical development. 


\section{MEETING SPECTRA}

\section{Specific learning and control disorder spectrum versus autism disorder spectrum}

Every form of atypical development is characterized by a straying-from-the-norm wiring of cerebral areas and atypical cerebral transmission; in other words, links do not function as efficiently or in other areas function too efficiently. Locations of deviations:

- hemisphere dominance

- contact between hemispheres

- adaptation system

- automatic processes

- cerebellum functioning

- executive functions of the prefrontal cortex

The following deviations manifest themselves:

- atypical memory functioning

- atypical information processing

- atypical perception

- atypical learning

- atypical motor coordination

- atypical executive functioning

Atypical kids are not only "disconnected kids" bit rather "atypically connected kids". More precisely, the specific learning and control disorder spectrum kids' wiring conforms to a lifestyle of several hundred thousand years ago, but it failed to transform perfectly to meet the requirements of the lifestyle of the last few thousands of years that are represented by the school. On the other hand, the autism spectrum kids' wiring has changed significantly to the new lifestyle and became almost the caricature of the breeder-farmer cognitive style.

In other words, there is a definite difference between two spectrums of atypical development, namely,

1. the specific learning-, attention- and hyperactivity disorders and

2. the autism disorders.

Williams and Casanova (2010) found local hyper-connectivity and long-range hypoconnectivity in autism, while the inverse arrangement of local hypo-connectivity and longrange hyper-connectivity in dyslexia, which confirms our assumption of the dichotomy.

However, the dichotomy can be questioned as besides the fact that it is not uncommon for dyslexia, dyscalculia, attention- or hyperactivity disorder to appear alongside autism disorder, brain physiological studies we presented earlier in this study, have shown that deviations in these cases can be found in the same areas.

Casanova and his colleagues' research results would be in contradiction with other research results and even the everyday experiences that show the kinship of all these atypicalities if 
we consider the brain wiring a static system. However, the brain is a constantly evolving, dynamic system, where instability can turn to over- and under-functioning, as well.

Too many connections or too few neither is optimal and need alternative ways. Having many gifted persons among those showing atypical development proves the advantage of the deviation from the considered optimum, a stable functioning.

\section{Extreme nomad and farmer characteristics}

Our view is that those on the specific learning /control spectrum are characterized by a nomad hunter-gatherer type of information processing, while those on the autism spectrum are the extremely good "breeders". These latter types are extremely good students for school. Although behaviour controlled by a brain overly biased towards school thinking may cause a lot of problems, still, a learner with high functioning autism disorder counts as ideal, or rather too ideal in the area of education, as opposed to the specific learning /control spectrum, which tends to manifest itself in achievement difficulties.

Students with autism are great at picking up detail and spitting back the information exactly how it was presented to them. This tendency can play out well in school for a while. The teacher can then believe that the student is understanding. The struggle for the student with autism in school is checking for a deep understanding. Are they making the connections that they should be making? Often the struggle is highlighted when given a bigger project, they may focus too intently on one aspect of the assignment and then struggle to complete the task or veer off track on something very loosely connected, missing the main overarching point to the assignment.

Mimicry is alive and well in the classroom. The teacher presents the material and the student is expected to give that information back to the teacher in the same form as it was presented. Students who can do this successfully often receive top marks.

The learners with the other spectrum disorder struggle to retain the details and specifics when presented with material in this way. However, they are often quite adept at taking that information and then synthesizing it or connecting it to something else. Yet, many classrooms do not focus on the critical thinking aspects of a subject. In mathematics for example, when the material is presented as a procedure that should be mimicked, they will struggle; yet if that same topic was taught through relational understanding, they would excel (Figure 3).

The mind characterized with specific learning / control disorder must understand first, and then they gradually learn the details irrespective of the level of intelligence. Yet, often in school when students struggle with a topic, there is a belief that because the student is struggling, they are not intelligent. This in combination with the cultural belief that memorization is easier than conceptual understanding, often prompts the teacher to break down the topic into little steps that the student is to memorize. This assumption of lack of intelligence and cultural belief in the ease of memorization and following instructions creates an environment for specific learning / control spectrum learners that is a downward spiral of constant challenge in school and contributes to their own belief in their lack of intelligence and normal behavior. 
Figure 3. Both under-functioning and over-functioning may cause difficulties in everyday activities. The extremity means the deviation.

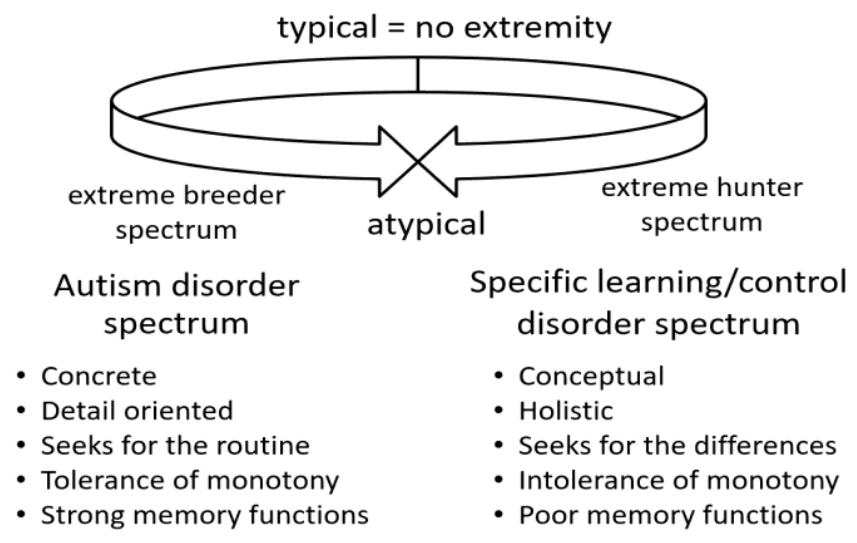

It should also be noted that Williams and Casanova (2010) did not find one particular region of the brain in all people with autism or all people with dyslexia to be more frequently affected than any other, hence the fingerprint analogy mentioned earlier. For example, it is a commonly held belief that all dyslexic people struggle with phonemic awareness. One would then expect that this area of the brain associated with phonemic awareness would be affected by wider brain folds in all dyslexic persons. Yet, Casanova and his colleagues did not find this to be the case. The area of the brain that deals with phonemic awareness were not more or less affected in all the dyslexic participants. Rather, they found there to be no pattern when it came to any one area as being more affected by wider folds than others, he just found variation throughout.

So, some areas within the dyslexic brain were more affected than others, but as a whole group, one area was not found to be consistently affected. This seems to support the extreme level of variation that is seen in how each student with these profiles presents areas of strength and weakness. Yet, there are still overarching characteristics that can be found within each of these groups.

Nielsen, Zielinski, Ferguson, Lainhart, and Anderson (2007) looking at over 1,000 brains discovered similar findings of variation, but in areas of brain activation rather than physiology. They found that none of their participants demonstrated preferential activation in one hemisphere over the other. Yet, there does seem to be evidence of left-handed people having more brain symmetry which would lead to better communication between the right and left sides. This connects well with Casanova's work around dyslexics having more connections from one hemisphere of the brain to the other and that dyslexics have a greater tendency to be left-handed.

Handedness and lateralization are heavily researched, and there is growing support from neuro-imaging studies that atypical or weak cerebral lateralization and an even less definitive handedness is associated with neurodevelopmental disorders such as autism, ADHD and dyslexia, (e.g., Preslar, Kushner, Marino \& Pearce, 2014; Knaus, Kamps \& Foundas, 2016; Simões, 2017).

The ambiguous data on many areas of atypical development is not a lack of proper investigation of the issue, but the sign of the diversity of atypical development. The research 
could identify rather tendencies, and two opposite spectra were found. Though opposite neurological deviations have been found in the background of specific learning /control and autism spectra, it is important to notice that the same areas of functioning show these differences in the case of the two types of atypical development.

Atypical brains are oscillating at the extremes in contrast to the brains considered typical. The direction of the deviation is not decisive, but both under- and over-functioning are on the extreme side, and that turns perceptions, reactions, and abilities atypical. Not only because of the extremity but also because of the unstable neurological functions.

The affected areas are the functions that have been needed in the change to agriculture some thousand years ago. The extremities show that an evolutionarily new neurological functioning is always vulnerable and easily variable.

\section{SUMMARY AND CONCLUSION}

Today's change in culture affects the maturation of the nervous system. We assume that this is the reason for the increased prevalence of atypical neurological development. According to our viewpoint atypical developments called dyslexia, dysgraphia, dyspraxia, dyscalculia, attention deficit, hyperactivity, and autism disorder are all indicators and indicate a change in the environment. Those with special developments are more sensitive than others to external influences, and as a result, deviations caused by agents affecting neurological development appear more quickly in their case.

There is relative recent work done in the area of cognitive neuroscience in which atypical neurological structure has been identified behind specific learning difficulties, ADHD and autism, and the researchers reported that the brain of a person with autism has more local connectivity than a "typical" brain structure but less long-range, whereas the dyslexic has fewer local but more long-range connectivity than is considered typical (Williams, Casanova, 2010). According to their results, these symptoms are at two ends of a continuum. This presence of more neural connections brings into question this idea of disorder and maturation. However, it also stirs up debate in that other researchers have found that autism with specific learning difficulties and ADHD is not a very rare combination and that learning difficulties, attention, hyperactivity, and autism spectrum disorders share some common neurological bases (e.g., Pauc, 2005; Moreno-De-Luca et al, 2013).

Research has suggested that several common features and co-morbidities support the idea to classify 3D / ADHD spectrum (which contains dyslexia, dysgraphia, dyspraxia, dyscalculia, attention deficit, hyperactivity disorders) and autism spectrum into one group. However, 3D / ADHD spectrum shows slightly different over-functions and under-functions at the different neural networks than in autism spectrum. Either over- or under-functioning can cause atypical development, the extremities and the instability of the neurological development is what group together the two rather opposite spectra.

Following along closer to a neurodiversity standpoint, rather than defining and naming these different brain organizations as disorders, could they instead be variations with muchneeded skills and abilities form our nomad hunter-gatherer style culture with tendencies that favour 3D / ADHD traits, and our agricultural-breeder style culture with tendencies that favour autistic traits. As we once again experience a culture shift, could a marrying of these 
atypical qualities be in increased demand in this new era of image and information overload rather than viewed as disorders?

Syndromes that are today called disorders, do not lead to performance problems in all cases, but rather could be interpreted as evolutionary answers to the environmental influences. $A$ new type of cognitive functioning is regarded by the environment as a disorder only as long as it is relatively rare in the population and impedes the attainment of usual performance. Once a phenomenon previously regarded as a disorder or an error is better suited to meet new challenges than older methods, it becomes an evolutionary advantage. And once the majority then also develops this cognitive functioning, which by this time manifests itself as an advantage, the error becomes the norm. This happened during the development of Homo sapiens. New activities supported new brain functioning and these brain functioning became very important in survival.

The different forms of exceptionality, which may not always be apparent, but often leads to special development, and which we have called atypical development (learning, attention, hyperactivity, and autism spectrum disorders) are becoming increasingly frequent, and who knows, maybe in a new culture that is what we need.

These specialties are not illnesses and cannot be cured, because they are exceptionalities in the maturation and functioning of the nervous system, which have had, and still can have, several advantages, as well as disadvantages, which is true of everything.

Human evolution did not stop at having become Homo sapiens. Humanity has been getting newer and newer designations to indicate different phases of its evolution: Homo oralis, Homo literalis, Homo typographicus, Homo interneticus (Goldhaber, 2004), and of course Homo informaticus, and according to Yuval Harari, in case we push forward our intervention into the evolution and lose control, the next step would be the Homo Deus, which would be the end of the history of the Homo sapiens (Harari, 2017).

Today, the environment and human culture are changing at a never-before-seen speed, which can best be identified in children's development. Children adapt to the world they are born into, and this in turn shapes their neurological functioning. We assume that most of the atypical developments are not disorders, but indicators. Their increasing prevalence is rooted in slower or different-from-typical development of neurological functions preparing the individual for literacy, behind which we can identify the cultural and physical-biological effects of the external environment. A deeper understanding of the atypical development may give insight into the culture-brain interaction during evolution.

Besides cultural factors, other environmental effects influencing the development of the nervous system may also play a role in the emergence of atypical development. Radiation or chemicals can easily affect the newer, less basic functions of the developing nervous system. COVID-19 pandemic causes a significant increase in the rate of atypical neurological development because viruses usually influence the development of the brain, and COVID-19 has an uncommonly large impact on the nervous system. This influence may not even be apparent but may significantly alter children's development.

A deviation in neurological functions can involve both under- and over-functioning. This can be assumed to be what is occurring in the case of an atypically developing brain. This is why the autism spectrum and what we call the 3D /ADHD spectrum are both atypical 
developments and may co-occur. Unstable functioning means that in some cases, underfunctioning may appear alongside over-functioning.

All cases of atypical development have more severe forms. The less apparent, and thereby unstable atypical development is a less obvious problem because it is not rooted in a severe injury or a developmental anomaly. This often, mixed atypical development is usually uncategorizable as a given diagnosis, as if it were atypical even for being an atypical development, indicating that they are not the ordinary well-known syndromes.

According to the person-environment fit theory (Caplan \& Harrison, 1993), a prerequisite for successful adaptation is the agreement and efficient interaction between personal characteristics (biological and psychological needs, values, goals, abilities, personality) and environmental characteristics (task, work, role expectations, cultural values, social environment).

The cultural change would not cause so many problems if we provided an environment suitable for the development of the nervous system typical of the altered circumstances. To achieve this task, however, we need to revise our views about "normality" and "typical development". 


\section{References}

Barkley, R.A., Koplowitz, S., Anderson, T., \& McMurray, M.B. (1997). Sense of time in children with ADHD: Effects of duration, distraction, and stimulant medication. Journal of the International Neuropsychological Society, 3, 359-369.

Bishop D.V.M. (2013). Cerebral asymmetry and language development: cause, correlate, or consequence? Science, 340. p. 1230-531.

Bishop, D.V.M., \& Snowling, M. J. (2004). Developmental Dyslexia and Specific Language Impairment: Same or Different? Psychological Bulletin, 130(6), 858-886.

Bomba M.D, \& Pang E.W. (2004). Cortical auditory evoked potentials in autism: A review. Int. J. Psychophysiol. 53: 161-169.

Boroditsky, L., \& Gaby, A. (2010). Remembrances of times east: absolute spatial representations of time in an Australian Aboriginal community. Psychological Science 21. 1635-1639.

Campbell-McBride, N. (2005). Gut and Psychology Syndrome. London, Medinform Publishing. Campbell-McBride, N. (2010). Gut and Psychology Syndrome: Natural Treatment for Autism, Dyspraxia, A.D.D., Dyslexia, A.D.H.D., Depression. London, Medinform Publishing.

Caplan, R. D., \& Harrison, R.V. (1993). Person-environment fit theory: Some history, recent developments, and future directions. Journal of Social Issues, 49, 253-275.

Casanova, M.F., Araque, J., Giedd, J., \& Rumsey, J.M. (2004). Reduced brain size and gyrification in the brains of dyslexic patients. Journal of Child Neurology, 19(4), 275-281.

Casanova, M. F., Buxhoeveden, D. P., Cohen, M., Switala, A. E., \& Roy, E. L. (2002). Minicolumnar pathology in dyslexia. Annals of Neurology, 52(1), 108-110.

Casanova, M.F., El-Baz, A.S., Giedd, J., Rumsey, J.M., \& Switala, A.E. (2010). Increased white matter gyral depth in dyslexia: implications for corticocortical connectivity. Journal of Autism and Developmental Disorders, 40(1), 21-29.

Cotgreave I.A. (2005). Biological stress responses to radio frequency electromagnetic radiation. Arch. Biochem. Biophys. 435(1):227-240.

Denckla, M.B., Rudel, R.G., Chapman, C., \& Krieger, J. (1985). Motor proficiency in dyslexic children with and without attentional disorders. Archives of Neurology, 42, 228-231.

Diamond, A. (2013). "Executive functions". Annual Review of Psychology. 64: 135-168.

Diamond, J., \& Bellwood, P. (2003). Farmers and Their Languages: The First Expansions. Science 25 Apr: Vol. 300, Issue 5619, pp. 597-603. DOI: 10.1126/science.1078208

Donald, M. (1991). Origins of the Modern Mind. Cambridge Mass.: Harvard University Press.

Eide, B., \& Eide, F. (2011). The dyslexic advantage: unlocking the hidden potential of the dyslexic brain. New York: Hudson Street Press.

Everett, D. L. (2005). Cultural constraints on grammar and cognition in Pirahă. Current Anthropology, 46, 621-646.

Everett, D. L. (2012). What does Pirahă grammar have to teach us about human language and the mind? WIREs Cognitive Science, 2012, 3: 555-563. 
Feingold, B. (1974). Why Your Child is Hyperactive. New York, Random House.

Ferreri, F., Curcio, G., Pasqualetti, P., et al. (2006). Mobile phone emissions and human brain excitability. Ann Neurol. 60(2):188-196.

Frank, M.C., Everett, D. L., Fedorenko, E., \& Gibson, E. (2008). Number as a cognitive technology: Evidence from Pirahă language and cognition. Cognition, 108 (2008) 819-824.

Gaby, A. (2011). "Where went "west"? The adoption of English communicative norms in Pormpuraaw talk about space." In. Australian Languages Workshop, 11th March, Stradbroke Island: University of Queensland

Goldhaber, M.H. (2004). The mentality of Homo interneticus: Some Ongian postulates. First Monday, Volume 9, Number 6-7, June.

Gordon, P. (2004). Numerical cognition without words: Evidence from Amazonia. Science, 306, 496-499.

Gowlett J.A. (2016). The discovery of fire by humans: a long and convoluted process. Philosophical transactions of the Royal Society of London. Series B, Biological Sciences, 371(1696), 20150164. https://doi.org/10.1098/rstb.2015.0164

Greenspan, S.I., \& Wieder, S. (1999). A functional developmental approach to autism spectrum disorders. Journal of the Association for Persons with Severe Handicaps, 24, 147161.

Gyarmathy É. (2009). Atipikus agy és a tehetség I. - Tehetség és a neurológia hátterű teljesítményzavarok valamint az Asperger szindróma. [The atypical brain and giftedness I. Giftedness, neurologically based achievement disorders and the Asperger syndrome] Pszichológia. Vol. 29, 4. 377-390.

Harari, Y.N. (2017). Homo Deus: A Brief History of Tomorrow. London: Vintage Healy, J.M. (1990). Endangered Minds: Why Our Children Don't Think. New York, Touchstone. Knaus, T.A, Kamps, J., \& Foundas, A.L. (2016). Handedness in Children With Autism Spectrum Disorder. Percept Mot Skills, Apr;122(2): 542-59.

Kouichi Yoshimasu, Chikako Kiyohara, Shigeki Takemura, Kunihiko Nakai (2014) A metaanalysis of the evidence on the impact of prenatal and early infancy exposures to mercury on autism and attention deficit/hyperactivity disorder in the childhood. NeuroToxicology, Volume 44, 121-131, ISSN 0161-813X,

Kurzweil, R. (2005). The Singularity is Near. New York: Viking Books.

Matsuzaki, H., Iwata, K., Manabe, T., \& Mori, N. (2012). Triggers for Autism: Genetic and Environmental Factors. Journal of Central Nervous System Disease.

Melillo R. (2009). Disconnected Kids: The Groundbreaking Brain Balance Program for Children with Autism, ADHD, Dyslexia, and Other Neurological Disorders. New York: Perigee Press.

Moreno-De-Luca, A., Myers, S.M., Challman, T.D., Moreno-De-Luca, D., \& Evans, D.W., Ledbetter, D.H. (2013). Developmental brain dysfunction: revival and expansion of old concepts based on new genetic evidence. Lancet Neurol, Apr;12(4):406-414 
Morton, J., \& Frith, U. (1995). Causal modelling: a structural approach to developmental psychopathology. In D. Cicchetti \& D. J. Cohen (Eds.). Manual of Developmental Psychopathology, Vol. 2, New York: Wiley, 274-298.

Mrazik, M., Dombrowski, S. C. (2010) The neurobiological foundations of giftedness. Roeper Review, 32(4), 224-234.

Nicolson, R. I. (2015). Positive Dyslexia. Rodin Books, Sheffield

Nicolson, R. I., \& Fawcett, A. J. (2007). Procedural learning difficulties: reuniting the developmental disorders? Trends in Neuroscience. 30, 135-141. doi:

10.1016/j.tins.2007.02.003

Nicolson, R. I. \& Fawcett, A. J. (1990). Automaticity: a new framework for dyslexia research? Cognition, 35, 159-182. doi: 10.1016/0010-0277(90)90013-a

Nicolson, R. I., \& Fawcett, A. J. (2019) Development of dyslexia: The delayed neural commitment framework. Frontiers in Behavioral Neuroscience, 13, 112.

Pauc, R. (2005). Comorbidity of dyslexia, dyspraxia, attention deficit disorder (ADD), attention deficit hyperactive disorder (ADHD), obsessive-compulsive disorder (OCD) and Tourette's syndrome in children: A prospective epidemiological study. Clinical Chiropractic, Volume 8, Issue 4, December, 189-198.

Pennington, B.F., Van Orden, G.C., Smith, S.D., Green, P.A., \& Haith, M.M. (1990). Phonological processing skills and deficits in adult dyslexics. Child Development, 61, pp. 1753-1778

Perrachione, T. K., Del Tufo, S. N., Winter, R. M., Murtagh, J., Cyr, A., Chang, P., Halverson, K., Ghosh, S.S., Christodoulou, J.A., \& Gabrieli, J. D. (2016). Dysfunction of rapid neural adaptation in dyslexia. Neuron, 92(6), 1383-1397.

Piek, J. P., Pitcher, T., c Hay, D. A. (1999). Motor coordination and kinaesthesis in boys with attention deficit-hyperactivity disorder. Developmental Medicine and Child Neurology, 41, 159-165.

Preslar, J., Kushner, H.I., Marino, L., \& Pearce, B. (2014). Autism, lateralisation, and handedness: A review of the literature and meta-analysis. Laterality: Asymmetries of Body, Brain and Cognition, Vol. 19, Issue 1.

Richardson, A.J., \& Ross, M.A. (2000). Fatty acid metabolism in neurodevelopmental disorder: a new perspective on associations between attention-deficit/hyperactivity disorder, dyslexia, dyspraxia and the autistic spectrum. Prostaglandins, Leukotrienes and Essential Fatty Acids. Volume 63, Issues 1-2, July, 1-9.

Rosa, D. (2010). Minimal Brain Dysfunction. In C.S. Clauss-Ehlers (Ed.). Encyclopedia of CrossCultural School Psychology. Springer, Boston, MA

Rowe, K. (1988). Synthetic Food Colorings and 'Hyperactivity': A Double-blind Crossover Study. Australian Paediatric Journal. 24, 143-147. p.

Schonfeld, I., Shaffer, D., \& Barmack, J. (1989). Neurological soft signs and school achievement: The mediating effects of sustained attention. Journal of Abnormal Child Psychology, 17, 575-596. 
Simões EN, Carvalho ALN, Schmidt SL. (2017) What does handedness reveal about ADHD? An analysis based on CPT performance. Res Dev Disabil. 2017 Jun;65:46-56. doi:

10.1016/j.ridd.2017.04.009. Epub 2017 Apr 23. PMID: 28433793.

Solomon, M. (2007). "Cognitive control in autism spectrum disorders". International Journal of Developmental Neuroscience. 26 (2): 239-47.

Vlachos, P., Andreou, E., Delliou, A., \& Agapitou, P. (2013). Dyslexia and hand preference in secondary school students. Psychol. Neurosci, Vol.6 no.1 Rio de Janeiro Jan./June.

http://dx.doi.org/10.3922/j.psns.2013.1.10

Ward, N.I. (2001) Hyperactivity and a Previous History of Antibiotic Usage. Nutrition Practitioner. Vol 3(3), 12.

Williams, E.L., \& Casanova, M. (2010). Autism and dyslexia: A spectrum of cognitive styles as defined by minicolumnar morphometry. Medical Hypotheses, 74, 59-62.

Zhou, K, \& Bowern C. (2015). Quantifying uncertainty in the phylogenetics of Australian numeral systems. Proceedings of Royal Society Publishing. B 282: 20151278. 